\title{
Versão Brasileira do Tool for Assessing Cultural Competence Training (TACCT): uma Matriz de Competência Cultural
}

\section{Brazilian Version of the Tool for Assessing Cultural Competence Training (TACCT): a Cultural Competence Model}

\author{
Janaina Carla Silva Oliveira Pimenta ${ }^{I}(\mathbb{D})$ \\ Talitha Nascimento Mendonça ${ }^{I}(\mathbb{D}$ \\ Igor Mychael Melo Ferreira ${ }^{I}(\mathbb{D}$ \\ Nathaniel Lucas Soares Lima ${ }^{I} \mathbb{D}$ \\ Bruno Simão Teixeira ${ }^{I}(\mathbb{D}$ \\ Rogério de Melo Costa Pinto ${ }^{I}$ (i) \\ Tânia Maria da Silva Mendonça ${ }^{I}$ D
}

\section{PALAVRAS-CHAVE}

- Educação Médica.

- Competência Cultural.

- Psicometria.

- Antropologia Médica.

Introdução: Este estudo teve como objetivos traduzir, adaptar e avaliar as propriedades psicométricas do Tool for Assessing Cultural Competence Training (TACCT) para o português falado no Brasil. Métodos: Os itens do TACCT foram adaptados transculturalmente por meio das etapas de tradução, reconciliação, retrotradução, revisão da retrotradução, revisores independentes, etapa Delphi, pré-teste e incorporação dos resultados do préteste no processo de tradução. A versão final do instrumento para a língua portuguesa foi autoaplicada em 320 estudantes de uma escola médica. Para verificar a precisão da escala, adotou-se a análise de confiabilidade. Utilizamos a análise fatorial confirmatória para avaliação da validade de construto e dimensionalidade do instrumento. Resultado: A versão final do TACCT mostrou-se adequada e teve sua validade e confiabilidade confirmadas. Conclusão: A versão brasileira do TACCT é válida e confiável e tem potencial para ser utilizada no processo de implantação, revisão ou aprimoramento de currículos de escolas médicas brasileiras. 


\section{ABSTRACT}

\section{KEYWORDS}

- Medical Education.

- Cultural Competence.

- Psychometrics.

- Medical Anthropolgy.
Introduction: To translate, adapt and evaluate the psychometric properties of the Tool for Assessing Cultural Competence Training (TACCT) to Brazilian Portuguese. Methods: The TACCT items were transculturally adapted through several stages, namely: translation, reconciliation, backtranslation, revision of backtranslation, independent reviews, Delphi stage, pretest, and incorporation of the pretest results into the translation process. The final Portuguese version of the instrument was self-applied by 320 medical students. The scale accuracy was verified using reliability analysis. We employed confirmatory factor analysis to assess the construct validity and instrument dimensionality. Results: The final version of the TACCT was adequate, and its validity and reliability were confirmed. Conclusion: The Brazilian Portuguese version of the TACCT is valid and reliable, and it can be used to implement, review, and improve new curriculum content of Brazilian medical schools.

Recebido em 5/7/20

Aceito em 3/10/20

\section{INTRODUÇÃO}

A literatura apresenta relatos sobre a relação de influência das disparidades étnicas e raciais na assistência médica ${ }^{1}$. Tais disparidades são movidas por preconceitos e estereótipos que, consequentemente, interferem de modo negativo na qualidade e nos resultados da assistência dos serviços prestados à população ${ }^{1}$.

Nesse contexto, retomamos os conceitos de competência cultural, que é um conjunto de comportamentos, atitudes e políticas congruentes presentes em um sistema, uma organização ou entre profissionais, que possibilita um trabalho eficaz em situações transculturais². Já a cultura refere-se aos padrões integrados do comportamento humano, que incluem a linguagem, os pensamentos, as ações, os costumes, as crenças e as instituições de grupos raciais, étnicos, sociais ou religiosos ${ }^{2}$. Portanto a demonstração da competência cultural implica a capacidade do/a profissional ou das instituições em lidar com as crenças, práticas e necessidades culturais apresentadas pelos pacientes e por suas comunidades ${ }^{3}$.

Há relatos, na literatura, de que o treinamento de médicos/as em competência cultural pode melhorar a comunicação entre prestadores/as de serviços de saúde e os/as pacientes ${ }^{4,5}$, além de aperfeiçoar o atendimento centrado no paciente ${ }^{6,7}$ e a qualidade da assistência em saúde ${ }^{8}$. No entanto, a revisão sistemática de Beach et al. ${ }^{9}$ mostrou que não havia métodos de ensino específicos com conteúdos eficazes para a construção da competência cultural.

Dessa forma, como o/a estudante de Medicina pode demonstrar a competência cultural - a compreensão sobre a maneira como pessoas de diversas culturas e crenças percebem a saúde e o processo da doença $(\text { disease })^{10}$ e respondem aos sintomas, à experiência da doença (illness $)^{10} \mathrm{e}$ a tratamentos? A busca pela resposta a esse questionamento foi motivação para que a Association of American Medical Colleges (AAMC) reunisse especialistas em competência cultural para desenvolver o Tool for Assessing Cultural Competence Training (TACCT) ${ }^{11}$. Essa ferramenta é composta por itens que representam os objetivos de aprendizagem dos domínios e as áreas de treinamento de competência cultural a serem necessariamente desenvolvidas pelos/as estudantes de Medicina ao longo do seu período de formação pré-internato ${ }^{11}$.

Um dos objetivos da AAMC é assegurar que o TACCT, seus domínios e itens sejam traduzidos e adaptados culturalmente nos idiomas mais falados em todo o mundo e que os itens traduzidos tenham propriedades psicométricas semelhantes às medidas originais ${ }^{12}$. Dessa forma, a globalização e a padronização de um conjunto comum de medidas direcionadas pelo TACCT permitirão a comparabilidade dos resultados de pesquisas e intervenções educacionais das escolas médicas em diversos países.

Existem diferentes recomendações metodológicas para a realização de tradução e adaptação cultural de instrumentos e questionários elaborados em idiomas diferentes da língua-alvo. Essas diretrizes seguem as normas internacionais de tradução de instrumentos, que propõem uma abordagem composta por múltiplas etapas, que têm como finalidade evitar versões inadequadas por não serem capazes de avaliar os construtos originais, comprometendo sua veracidade ${ }^{13,14}$. A utilização de uma metodologia rigorosa, minuciosamente documentada e com sucessivas etapas visa garantir a qualidade do processo de tradução e adaptação cultural, sendo considerada a melhor forma de atingir as equivalências pretendidas entre as versões original e traduzida ${ }^{13,14}$.

Nesse sentido, o estudo de Boardman ${ }^{12}$ delineou os processos metodológicos apropriados para garantir as propriedades psicométricas do TACCT (validade e confiabilidade). Portanto, este estudo teve com objetivos traduzir, adaptar transculturalmente e avaliar psicometricamente o TACCT para o português falado no Brasil, para que ele possa ser disponibilizado como proposta de uma matriz da competência cultural nos currículos das escolas médicas brasileiras.

\section{MÉTODOS}

Estudo e participantes

O estudo de adaptação transcultural e validação psicométrica foi realizado com estudantes da fase pré-clínica do curso de Medicina da Universidade Federal de Uberlândia (UFU), de Minas Gerais, e aprovado pelo Comitê de Ética em Pesquisa dessa instituição ( $\mathrm{n}^{\circ}$ 2.088.832). $\mathrm{O}$ estudo foi realizado após permissão da AAMC. Os/as estudantes assinaram o Termo de Consentimento Livre e Esclarecido que permitiu a resposta do instrumento e gravações de áudio na etapa do pré-teste.

\section{Tamanho da amostra}

Para o pré-teste optamos, por utilizar uma média de aplicação de cinco itens para cada estudante ${ }^{15}$. Como a versão de competências do TACCT apresenta 67 itens, contamos com a participação de dois/duas estudantes, sendo o/a representante de turma e seu/sua colega substituto/a de cada um dos oito períodos pré-clínicos. Portanto, a amostra total da 
etapa de pré-teste contou com 16 participantes. Esses/as representantes seriam excluídos caso desistissem de continuar participando.

Para o processo de análise estrutural do TACCT, no qual utilizamos a análise fatorial $(\mathrm{AF})$, baseamo-nos nas recomendações de Clark e Watson ${ }^{16}$ que recomendam o uso de amostras apropriadamente heterogêneas que representem a população-alvo. Ademais, Comrey e Lee ${ }^{17}$ classificam, em relação a esse tipo de análise, amostras de 50 como muito inferiores, de 100 como inferiores, de 200 como razoáveis, de 300 como boas, de 500 como muito boas e de mil ou mais como excelentes. Portanto, utilizamos por sorteio 40 estudantes representantes de cada um dos oito períodos da fase pré-clínica do curso de Medicina, que concordassem em participar do estudo, totalizando uma amostra de 320 participantes. Caso esses/as estudantes desejassem se retirar após serem incluídos/as, seria feito novo sorteio.

\section{Instrumento}

O TACCT é uma matriz desenvolvida pela AAMC para avaliar o treinamento de competência cultural nas escolas de Medicina. Essa matriz é apresentada por meio dos componentes "visão geral" e "competências". O formato de "visão geral" apresenta cinco domínios e 22 itens ${ }^{11}$. Os domínios são os seguintes: "fundamentação, contexto e definição", que tratam dos conceitos de raça, etnia e cultura (três itens); "aspectos-chave da competência cultural", que aborda as tradições e estratégias de cura dos pacientes (quatro itens); "compreensão do impacto dos estereótipos nas decisões médicas", que avalia o preconceito, a discriminação e o racismo (três itens); "disparidades e fatores influenciadores da saúde", que aborda a história da discriminação de cuidados de saúde e epidemiologia das disparidades em saúde (cinco itens); e "intercâmbio cultural e habilidades clínicas", que enfatiza conceitos de diferentes valores, culturas e crenças (sete itens) ${ }^{11}$. Cada um desses domínios é avaliado no componente das competências do aprendizado, considerando os componentes de conhecimento, habilidade e atitude. Portanto, a matriz por competências contempla os 67 itens ${ }^{11}$.

O TACCT finaliza-se em 67 itens com respostas dicotômicas que, no caso de uma competência cultural que não foi abordada, o/a respondente replica como "não ofertado" (NO), e a esse item atribui-se valor 0 àqueles que foram abordados e adequadamente discutidos, atribui-se o escore 1 ("sim"). Posteriormente, é feita uma soma da frequência das respostas "sim", e uma média é calculada dividindo-se o resultado total pelo número de possibilidades de respostas ${ }^{11}$. A fim de uma análise mais precisa, também são calculados os escores dos componentes individuais de conhecimento, habilidade e atitude ${ }^{11}$.

Métodos analíticos dos dados qualitativos e quantitativos

Os apêndices 1 e 2 estão disponibilizados como material suplementar para que o leitor possa seguir de maneira sumarizada a nossa proposta de metodologia de análise de dados.

Equivalência semântica

\section{Adaptação transcultural}

Essa adaptação foi realizada de acordo com as diretrizes internacionais $^{18,19}$ (Apêndice 1). Na primeira etapa - tradução inicial -, dois tradutores nativos no português brasileiro e experts em inglês produziram duas traduções independentes do instrumento, do inglês para o português do Brasil. Na segunda etapa - reconciliação -, uma profissional de saúde bilíngue, nativa no português brasileiro, realizou a conciliação das traduções anteriores. Na terceira etapa - retrotradução -, um tradutor nativo no inglês norte-americano e fluente em português traduziu a versão reconciliada de volta para a língua original. Na quarta etapa, uma coordenadora de linguagem fez comentários sobre a versão reversa, que foi encaminhada para os desenvolvedores. Na quinta etapa, a metodologia Delphi, expressões, sentenças ou palavras identificadas como sem equivalência semântica, idiomática, experimental ou conceitual em qualquer etapa do processo de tradução foram encaminhadas para a coordenadora de linguagem, que avaliou, com cinco revisores/as bilíngues nativos/as do português brasileiro, com experiência no processo de tradução de medidas de resultados, todas as etapas anteriores para escolher a melhor opção traduzida para o instrumento. A técnica Delphi modificada ${ }^{20,21}$ foi utilizada para se chegar a um consenso entre os/as revisores/ as em rodadas eletrônicas iterativas organizadas por dois coordenadores de pesquisa, por meio de questionários semiestruturados no site Google Forms. Para o recrutamento dos/as especialistas, convidamos quatro profissionais da saúde, com experiência comprovada - por meio de publicações na literatura científica - em tradução, adaptação transcultural e validação de instrumentos de medida, além do domínio de educação médica e competência cultural.

Aplicamos a versão traduzida e adaptada transculturalmente para a fase de pré-teste com a participação de dois/duas estudantes de cada um dos oito períodos pré-clínicos. Durante a aplicação dos itens, observamos se eles/as estavam utilizando espontaneamente a sondagem verbal e o parafraseamento dos itens, a fim de validar as equivalências semântica, conceitual, operacional e funcional ou propor mudanças consistentes com os itens originais ${ }^{22,23}$. Mostramos essas etapas no Apêndice 1 (Equivalência semântica - adaptação transcultural do TACCT).

\section{Análise psicométrica}

\section{Estatística descritiva}

\section{a. Análise de itens}

Utilizamos a estatística descritiva para caracterizar a população da fase de validação do estudo, o que foi feito por meio de um questionário semiestruturado. Adotamos o software SPSS 24.0 e RStudio 3.6.1. Consideramos um nível de significância de $5 \%$.

Padrões e frequência de dados ausentes foram examinados para identificar a probabilidade de padrões sistemáticos ou aleatórios e não deveriam ultrapassar $20 \%$ do total ${ }^{24}$.

Não se forneceu uma explicação detalhada do conteúdo de itens individuais do TACCT no momento de sua aplicação. Os/as entrevistados/ as foram solicitados/as a interpretar cada item pelo valor nominal como o entenderam e não verificar os itens que não entenderam ou que não foram ensinados no currículo exigido. Nosso objetivo foi o entendimento linguístico do instrumento.

\section{Análise da escala}

\section{Confiabilidade}

Para fornecermos informações relacionadas à homogeneidade do conjunto de itens do TACCT, utilizamos a confiabilidade da consistência interna calculada no software SPSS 24.0. Nela, consideramos o coeficiente para medidas dicotômicas de Kuder-Richardson (KR-20) que, embora seja semelhante ao coeficiente alfa de Cronbach, é específico para respostas

REVISTA BRASILEIRA DE EDUCAÇÃo MÉDICA

3 44 (4) : e168; 2020 
binárias, com valores acima de 0,70 como mínimo aceito e ideal para 0,80, referentes às medidas do nível de grupo ${ }^{25}$.

\section{a. Validade de construto}

Utilizamos a análise fatorial confirmatória (AFC) e assumimos que todos os itens do TACCT possuíam carga fatorial de apenas um fator, o que representa uma estratégia confirmatória da modelagem de equações estruturais usada para explicar a relação entre os itens e o fator ou construto geral associado ao instrumento avaliado $^{26}$. O método de estimação que utilizamos foi o dos mínimos quadrados ponderados com ajustes para média e variância (weighted least square mean and variance adjusted - WLSMV) em função da natureza categórica dos nossos dados ${ }^{27}$. Os índices de ajuste analisados foram: índice de ajuste comparativo (comparative fit index - CFI; ideal quando $>0,90$ ), aproximação do erro quadrático médio da raiz (root mean square error of approximation - RMSEA; $<0,08$ ajuste adequado, < 0,06 ajuste satisfatório) e o índice de Tucker-Lewis (TLI; ideal quando > $0,90)$ e weighted root mean square residual (WRMR; $<0,9$ adequado) ${ }^{26}$. Para esta análise, utilizamos os pacotes Polycor, MASS, GPA rotation e Parallel do software R que são específicos para AFC de dados binários.

\section{RESULTADOS}

Equivalência semântica: adaptação transcultural

\section{Tradução inicial}

O termo clinicians (domínio I, item C) apresentou discrepância entre os dois tradutores brasileiros. $\mathrm{O}$ tradutor 1 considerou como "profissionais da saúde", e o tradutor 2, como "clínicos".

A palavra bias, presente nos componentes "visão geral" (domínio III, item B) e "componentes específicos" (domínio I, item A1; domínio III, itens K2, K3, de S1 a S3, de A1 a A5; domínio IV, item S4; e domínio V, itens S5 e A2), também teve divergência nas traduções. Um dos tradutores a considerou como "enviesamento", e o outro, como "preconceitos".

Nos componentes "visão geral" (domínio IV, item A), houve discrepância, entre os tradutores brasileiros, sobre a palavra history, que foi traduzida como "história" e "histórico". Além disso, ambos os tradutores mantiveram a expressão Healthy People 2010, como na versão original.

\section{Reconciliação}

O termo clinicians foi considerado como "profissionais da saúde", e bias, como enviesamento. Quanto à palavra history, houve um consenso de que a tradução mais adequada seria "história".

\section{Retrotradução}

No componente "visão geral", no domínio III, item C, da versão original, a expressão effects of stereotyping foi transformada em consequences of stereotyping. Nos componentes específicos pré-clínicos, no domínio
II, item A2, da ferramenta original, a expressão listen nonjudgmentally to health beliefs foi considerada, na versão retrotraduzida, como listen to health beliefs without bias.

Comparação entre a versão retrotraduzida e a versão original

A coordenadora de linguagem sugeriu que os itens clinicians e bias, bem como as expressões effects of stereotyping/consequences of stereotyping e listen nonjudgmentally to health beliefs/listen to health beliefs without bias", fossem rediscutidos na etapa Delphi.

\section{Delphi}

Os/as revisores/as independentes foram unânimes ao considerarem que, na versão reconciliada, a expressão "profissionais da saúde" não condizia especificamente ao contexto da ferramenta, que é avaliar a competência cultural nos currículos médicos. Dessa forma, julgaram que o termo adequado à versão pré-teste de clinicianspara a cultura brasileira seria médicos/as. Os/as avaliadores/as da etapa Delphi foram unânimes ao considerarem que a palavra bias deveria ser adaptada para "preconceito".

\section{Versão pré-final}

A coordenadora de linguagem avaliou a versão que seria aplicada no pré-teste.

\section{Pré-teste e revisão final}

Participaram da etapa de pré-teste 16 estudantes, dois/duas de cada um dos oito períodos pré-clínicos do curso de Medicina da Faculdade de Medicina da UFU (Famed-UFU). O teste do qui-quadrado para análise de proporção entre a variáveis não mostrou significância $(\mathrm{p}<0,005)$ para: os gêneros feminino $(9 ; 56,3,0 \%)$ e masculino $(7 ; 43,8 \%)$, autodeclarados como pardos $(9 ; 56,3 \%)$, pretos $(4 ; 25 \%)$ e brancos $(3 ; 18,8 \%)$, com média de idade de 21,9 $\pm 0,53$ anos (mínimo de 18 e máximo de 25 anos), todos/ as solteiros/as e sem filhos; residentes com a família $(8 ; 50 \%)$, com amigos $(31,3 \%)$ e sozinhos/as (3; 18,8\%); católicos/as $(5 ; 31,3 \%)$, evangélicos/as ( $4 ; 25 \%)$, espíritas $(4 ; 25 \%)$, sem religião $(2 ; 12,5 \%)$ e religião indefinida ( 1 ; $6,3 \%)$; não praticantes da religião (10;62,5\%), com doenças crônicas e uso de medicação $(9 ; 56,3 \%)$. Houve significância pelo teste do qui-quadrado ( $\mathrm{p}<0,005)$ para a declaração de renda: $75 \%$ dos participantes (12) se recusaram informar esse dado.

Todos/as os/as participantes da fase de pré-teste consideraram os itens não ofensivos, importantes e de fácil entendimento. Todos/as os/as participantes concordaram, durante os dois grupos focais, que a expressão Healthy People 2010 deveria ser substituída por "Datasus", a fim de adaptar a ferramenta à realidade brasileira, pois a expressão original poderia tornar o item incompreensível. A versão do pré-teste e a versão final do TACCT estão disponíveis integralmente nos quadros 1 e 2. 


\begin{tabular}{|c|c|c|}
\hline \multicolumn{3}{|c|}{$\begin{array}{c}\text { Quadro } 1 \\
\text { Visão geral do TACCT }\end{array}$} \\
\hline Domínio & Versão norte-americana & Versão brasileira \\
\hline I - Fundamentação, contexto e definição & $\begin{array}{l}\text { A. Definition of cultural competence } \\
\text { B. Definitions of race, ethnicity, and culture } \\
\text { C. Clinicians' self assesment and reflection }\end{array}$ & $\begin{array}{l}\text { A. Definição de competência cultural } \\
\text { B. Definições de raça, etnia e cultura } \\
\text { C. Autoavaliação e reflexão médica }\end{array}$ \\
\hline II - Aspectos-chave da competência cultural & $\begin{array}{l}\text { A. Epidemiology of population health } \\
\text { B. Patients' healing traditions and systems } \\
\text { C. Institutional cultural issues } \\
\text { D. History of the patient }\end{array}$ & $\begin{array}{l}\text { A. Epidemiologia da saúde populacional } \\
\text { B. Tradições e sistemas de cura de pacientes } \\
\text { C. Questões culturais institucionais } \\
\text { D. História do paciente }\end{array}$ \\
\hline $\begin{array}{l}\text { III - Compreendendo o impacto do estereótipo } \\
\text { na tomada de decisão médica }\end{array}$ & $\begin{array}{l}\text { A. History of stereotyping } \\
\text { B. Bias, discrimination, and racism } \\
\text { C. Effects of stereotyping }\end{array}$ & $\begin{array}{l}\text { A. A história do estereótipo } \\
\text { B. Preconceitos, discriminação e racismo } \\
\text { C. Consequências da estereotipagem }\end{array}$ \\
\hline $\begin{array}{c}\text { IV - Disparidades em saúde e fatores que a } \\
\text { influenciam }\end{array}$ & $\begin{array}{l}\text { A. History of health-care discrimination } \\
\text { B. Epidemiology of health-care disparities } \\
\text { C. Factors underlying health-care disparities } \\
\text { D. Demographic patterns of disparities } \\
\text { E. Collaborating with communities }\end{array}$ & $\begin{array}{l}\text { A. História da discriminação em saúde } \\
\text { B. Epidemiologia das disparidades com cuidados de saúde } \\
\text { C. Fatores subjacentes às disparidades nos cuidados com a saúde } \\
\text { D. Padrões demográficos das disparidades } \\
\text { E. Colaborando com comunidades }\end{array}$ \\
\hline V - Habilidades clínicas transculturais & $\begin{array}{l}\text { A. Differing values, cultures, and beliefs } \\
\text { B. Dealing with hostility/discomfort } \\
\text { C. Eliciting a social and medical history } \\
\text { D. Communication skills } \\
\text { E. Working with interpreters } \\
\text { F. Negotiating and problem-solving skills } \\
\text { G. Diagnosis and patient-adherence skills }\end{array}$ & $\begin{array}{l}\text { A. Diferenciando valores, culturas e crenças } \\
\text { B. Lidando com a hostilidade e o desconforto } \\
\text { C. Elicitando o histórico social e médico } \\
\text { D. Habilidades de comunicação } \\
\text { E. Trabalhando com intérpretes } \\
\text { F. Habilidades de negociação e de resolução de problemas } \\
\text { G. Habilidades para o diagnóstico e a adesão de pacientes. }\end{array}$ \\
\hline
\end{tabular}

Fonte: Elaborado pelos autores.

\section{Quadro 2}

Versão por Competências do TACCT

Domínio

Versão norte-americana

K1. Define race, ethnicity, and culture.

K2. Identify how race and culture relate to health.

K3. Identify patterns of national data on disparities.

K4. Describe health data with immigration context.

S1. Discuss race \& culture in the medical interview.

S2. Use physician assessment tools.

S3. Concretize epidemiology of disparities.

A1. Describe own cultural background and biases

A2. Value link between communication \& care.

A3. Value importance of diversity in health care.

K1. Describe historical models of health beliefs.

K2. Recognize patients' healing traditions \& beliefs.

K3. Describe cross-cultural communication challenges.

K4. Demonstrate knowledge of epidemiology.

\section{Versão brasileira}

C1. Definir raça, etnia e cultura.

C 2. Identificar como raça e cultura relacionam-se à saúde.

C 3. Identificar padrões de dados nacionais sobre disparidades.

C 4. Descrever os dados de saúde no contexto da imigração.

H1. Discutir raça, etnia e cultura numa entrevista médica.

H 2. Utilizar ferramentas de avaliação médica.

H 3. Constituir a epidemiologia de disparidades

A1. Descrever o próprio histórico cultural e preconceito.

A2. Valorizar a conexão entre comunicação e cuidado.

A3. Valorizar a importância da diversidade no cuidado em saúde.

C1. Descrever os modelos históricos de crenças em saúde.

C2. Reconhecer tradições e crenças de cura dos pacientes.

C3. Descrever desafios da comunicação transcultural.

C4. Demonstrar conhecimento sobre epidemiologia. 


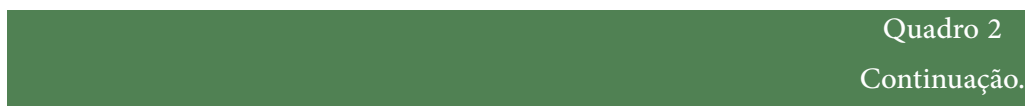

Domínio

Versão norte-americana

K5. Describe population health variability factors.

S1. Outline a framework to assess communities.

S2. Ask questions to elicit patient preferences.

S3. Elicit information in family-centered context.

Aspectos-chave da competência cultural

S4. Collaborate with communities to address needs.

S5. Recognize institutional cultural issues.

A1. Exhibit comfort when discussing cultural issues.

A2. Listen nonjudgmentally to health beliefs.

A3. Value \& address social determinants of health.

A4. Value curiosity, empathy, and respect.

K1. Describe social cognitive factors.

K2. Identify physician bias and stereotyping.

K3. Recognize physicians' own potential for biases.

K4. Describe the physician-patient power imbalance.

K5. Describe physician effect on health disparities.

K6. Describe community partnering strategies.

S1. Demonstrate strategies to address/reduce bias.

S2. Describe strategies to reduce physician biases.

Impacto do estereótipo na tomada de decisão médica

O impacto do estereótipo na tomada de decisão médica
S3. Show strategies to address bias in others.

S4. Engage in reflection about own beliefs.

S5. Use reflective practices when in patient care.

S6. Gather and use local data as in HP2010.

A1. Identify physician biases that affect clinical care.

A2. Recognize how physician biases impact care.

A3. Describe potential ways to address bias.

A4. Value the importance of bias on decision-making.

A5. Value the need to address personal bias.

K1. Describe factors that impact health.

K2. Discuss social determinants on health.

K3. Describe systemic \& medical encounter issues.

K4. Identify and discuss key areas of disparities.

K5. Describe elements of community experiences.

K6. Discuss barriers to eliminating health disparities.

S1. Critically appraise literature on disparities.

S2. Describe methods to identify community leaders.

S3. Propose a community-based health intervention.

\section{Versão brasileira}

C5. Descrever os fatores de variabilidade da saúde da população.

H1. Delinear um modelo para avaliar comunidades.

H2. Fazer perguntas para identificar preferências do paciente.

H3. Extrair informações relacionadas ao contexto familiar.

H4. Obter informações relacionadas ao contexto familiar para o mapeamento de necessidades.

H5. Reconhecer questões culturais institucionais.

A1. Demonstrar conforto ao discutir questões culturais.

A2. Ouvir sem julgamento sobre crenças em saúde.

A3. Valorizar e abordar determinantes sociais de saúde.

A4. Valorizar a curiosidade, a empatia e o respeito.

C1. Descrever os fatores cognitivos sociais.

C2. Identificar enviesamento e estereotipagem médica.

C3. Reconhecer o potencial do médico aos próprios preconceitos.

C4. Descrever o desequilíbrio na relação de poder entre médico e paciente.

C5. Descrever a influência de médicos nas disparidades em saúde.

C6. Descrever as estratégias de parceria com a comunidade.

H1. Demonstrar ter estratégias para abordar/reduzir preconceitos dos outros.

H2. Descrever estratégias para reduzir preconceitos de médicos.

H3. Demonstrar ter estratégias para abordar preconceitos nos outros.

H4. Refletir sobre suas próprias crenças.

H5. Utilizar práticas reflexivas para o cuidado do paciente.

H6. Coletar e utilizar dados do Datasus.

A1. Identificar preconceitos médicos que afetam cuidados clínicos.

A2. Reconhecer como preconceitos médicos influenciam o cuidado.

A3. Descrever formas de lidar com o preconceito.

A4. Atribuir valor à importância do preconceito na tomada de decisão.

A5. Valorizar a necessidade de lidar com seus próprios preconceitos.

C1. Descrever fatores que impactam a saúde.

C2. Discutir determinantes sociais sobre a saúde.

C3. Descrever questões de sistêmicas e médicas.

C4. Identificar e discutir as principais áreas de disparidades.

C5. Descrever elementos sobre experiências comunitárias.

C6. Discutir barreiras à eliminação das desigualdades em saúde.

H1. Avaliar criticamente a literatura sobre desigualdades.

H2. Descrever métodos para identificar líderes comunitários.

H3. Propor uma intervenção de saúde baseada na comunidade. 


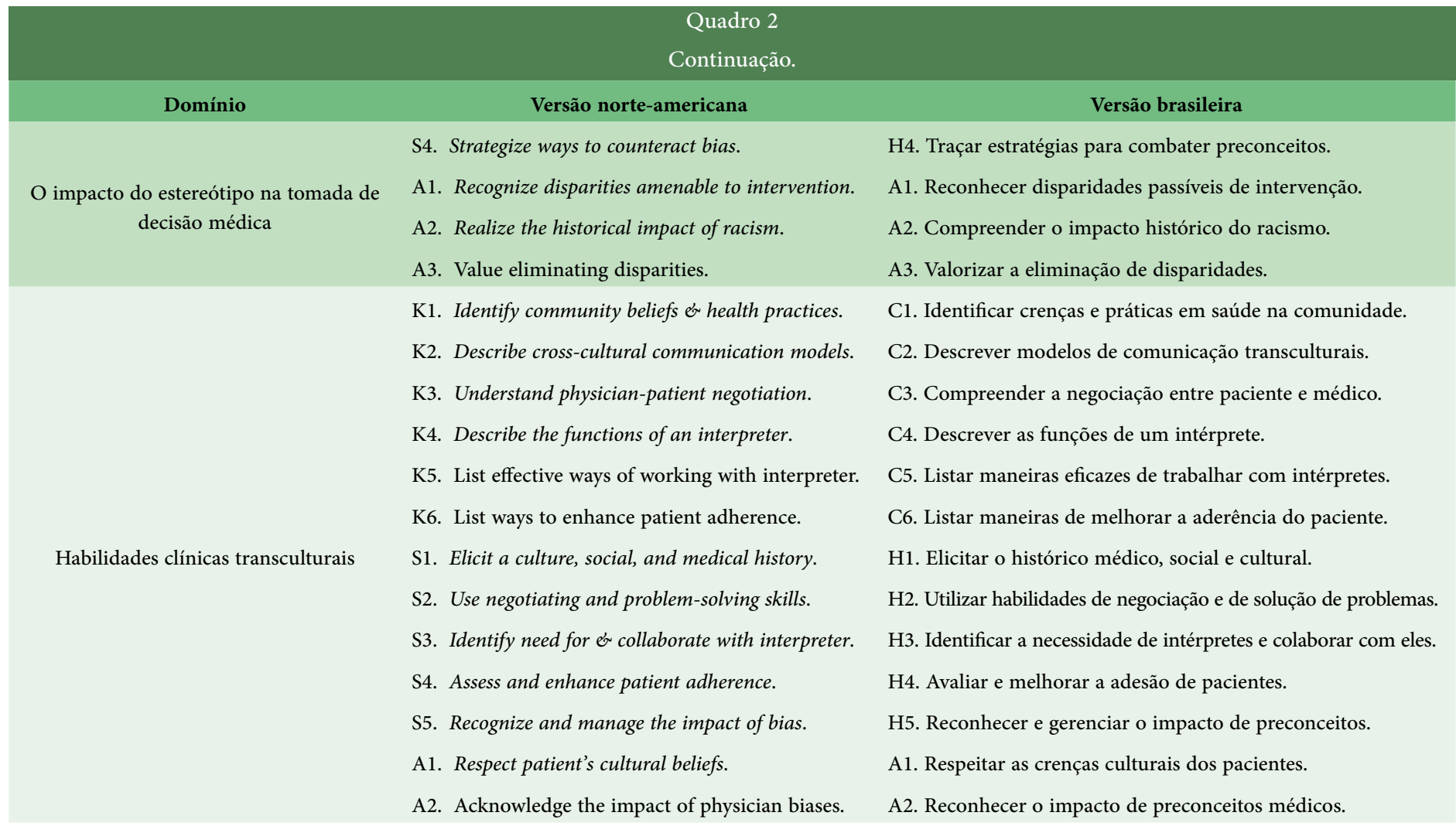

Fonte: Elaborado pelos autores.

\section{Validação psicométrica}

Participaram dessa etapa 320 estudantes, representantes dos oito períodos pré-clínicos do curso de Medicina da UFU, e a Tabela 1 mostra suas principais características.

Consistência interna do item

A consistência interna do TAACT apresentou o KR-20 de 0,95. Esse resultado mostra que grande parte da variação dos domínios está sendo explicada pelos itens que os compõem.

\section{Validade de construto}

A estrutura fatorial do TACCT foi confirmada por meio de uma AFC. Os resultados de ajuste foram favoráveis à sua estrutura fatorial $\left(\mathrm{X}^{2}(\mathrm{GL}=2211)=13513,75 ; \mathrm{p}<0,001\right.$; CFI $=0,88$; TLI = 0,529; RMSEA $=0,06$. A Figura 1 apresenta os cinco construtos do TAACT, as correlações moderadas entre os construtos $(0,4$ e 0,5$)$ e as cargas fatoriais acima de 0,3 .

\section{DISCUSSÃO}

Realizamos um estudo transversal metodológico com o objetivo de disponibilizar a versão norte-americana do TACCT para o português falado no Brasil e optamos por manter a mesma sigla da língua inglesa. Para isso, o instrumento foi traduzido e adaptado transculturalmente e teve suas propriedades psicométricas de validade e confiabilidade confirmadas pela participação de estudantes dos períodos antecedentes à fase de internato do curso de Medicina da Famed-UFU.

\begin{tabular}{|c|c|c|}
\hline \multicolumn{3}{|c|}{$\begin{array}{c}\text { Características dos/as participantes da fase de validação das } \\
\text { propriedades psicométricas do TACCT }\end{array}$} \\
\hline Variável & Medida & $\mathrm{X}^{2}$ (GL); P- valor \\
\hline Idade, média (DP) & $22,2( \pm 3,7)$ & na \\
\hline \multicolumn{3}{|l|}{ Sexo, n (\%) } \\
\hline Feminino & $152(47,9)$ & $0,54(1) ; 0,465$ \\
\hline Masculino & $165(52,1)$ & \\
\hline $\begin{array}{l}\text { Renda familiar, em } \\
\text { salário mínimo, n (\%) }\end{array}$ & & $571,21(5) ; 0,000$ \\
\hline Não informada & $206(65)$ & \\
\hline Até 1 & $3(0,9)$ & \\
\hline De 1 a 2 & $16(5)$ & \\
\hline De $3 a 4$ & $39(12,3)$ & \\
\hline Acima de 4 & $53(16,8)$ & \\
\hline \multicolumn{3}{|l|}{ Cor, n (\%) } \\
\hline Pardo & $120(37,9)$ & $418,5(4) ; 0,000$ \\
\hline Preto & $13(4,1)$ & \\
\hline Amarelo & $4(1,3)$ & \\
\hline Branca & $179(56,5)$ & \\
\hline Indígena & $1(0,3)$ & \\
\hline
\end{tabular}

Continuação.. 


\begin{tabular}{|c|c|c|}
\hline \multicolumn{3}{|c|}{ Tabela 1} \\
\hline \multicolumn{3}{|c|}{ Continuação } \\
\hline Variável & Medida & $\mathrm{X}^{2}$ (GL); P- valor \\
\hline \multicolumn{3}{|l|}{ Estado civil, n (\%) } \\
\hline Solteiro/a & $306(96,5)$ & $865,58(3) ; 0,000$ \\
\hline Casado/a & $9(2,8)$ & \\
\hline Divorciado/a & $2(0,7)$ & \\
\hline \multicolumn{3}{|l|}{ Filhos, n (\%) } \\
\hline Nenhum & $311(98,1)$ & 598,59 (2); 0,000 \\
\hline$U m$ & $5(1,6)$ & \\
\hline Dois & $1(0,3)$ & \\
\hline \multicolumn{3}{|l|}{ Moradia, n (\%) } \\
\hline Sozinho/a & $89(28,2)$ & $341,24(6) ; 0,000$ \\
\hline Pais & $126(39,7)$ & \\
\hline Cônjuge & $9(2,8)$ & \\
\hline Parentes & $13(4,1)$ & \\
\hline Amigos & $77(24,3)$ & \\
\hline Alojamento estudantil & $3(0,9)$ & \\
\hline \multicolumn{3}{|l|}{ Religião, n (\%) } \\
\hline Católica & $103(32,5)$ & $161,45(6) ; 0,000$ \\
\hline Evangélica & $57(18)$ & \\
\hline Espírita & $42(13,2)$ & \\
\hline Ateu/ateia & $68(21,5)$ & \\
\hline Indefinida & $37(11,7)$ & \\
\hline Não declarada & $10(3,1)$ & \\
\hline \multicolumn{3}{|l|}{ Prática religiosa, n (\%) } \\
\hline Sim & $119(37,5)$ & $19,69(1) ; 0,000$ \\
\hline Não & $198(62,5)$ & \\
\hline Doença crônica, n (\%) & $10(66,7)$ & \\
\hline Sim & $41(12,9)$ & $174,21(1) ; 0,000$ \\
\hline Não & $276(87,1)$ & \\
\hline
\end{tabular}

Fonte: Elaborada pelos autores.

O TACCT é uma matriz importante para avaliar quais áreas da competência cultural representadas nesse instrumento foram ensinadas durante os primeiros quatro anos da Faculdade de Medicina. Esse instrumento pode servir como disparador para que as escolas médicas brasileiras possam, por meio da proposta dessa matriz, aprimorar seus currículos, a fim de que os/as estudantes possam realmente alcançar seu aprendizado relacionado à competência cultural, com o objetivo de eliminar as disparidades raciais e étnicas na área da saúde. Isso se faz necessário para que se atenda ao princípio constitucional e doutrinário do Sistema Único de Saúde (SUS) de universalidade do acesso à saúde, que prevê, a toda a população imigrante, o direito à atenção à saúde de maneira humanizada e qualificada ${ }^{28}$. Nesse sentido, destacamos que a atenção primária à saúde (APS) é um cenário possível para o

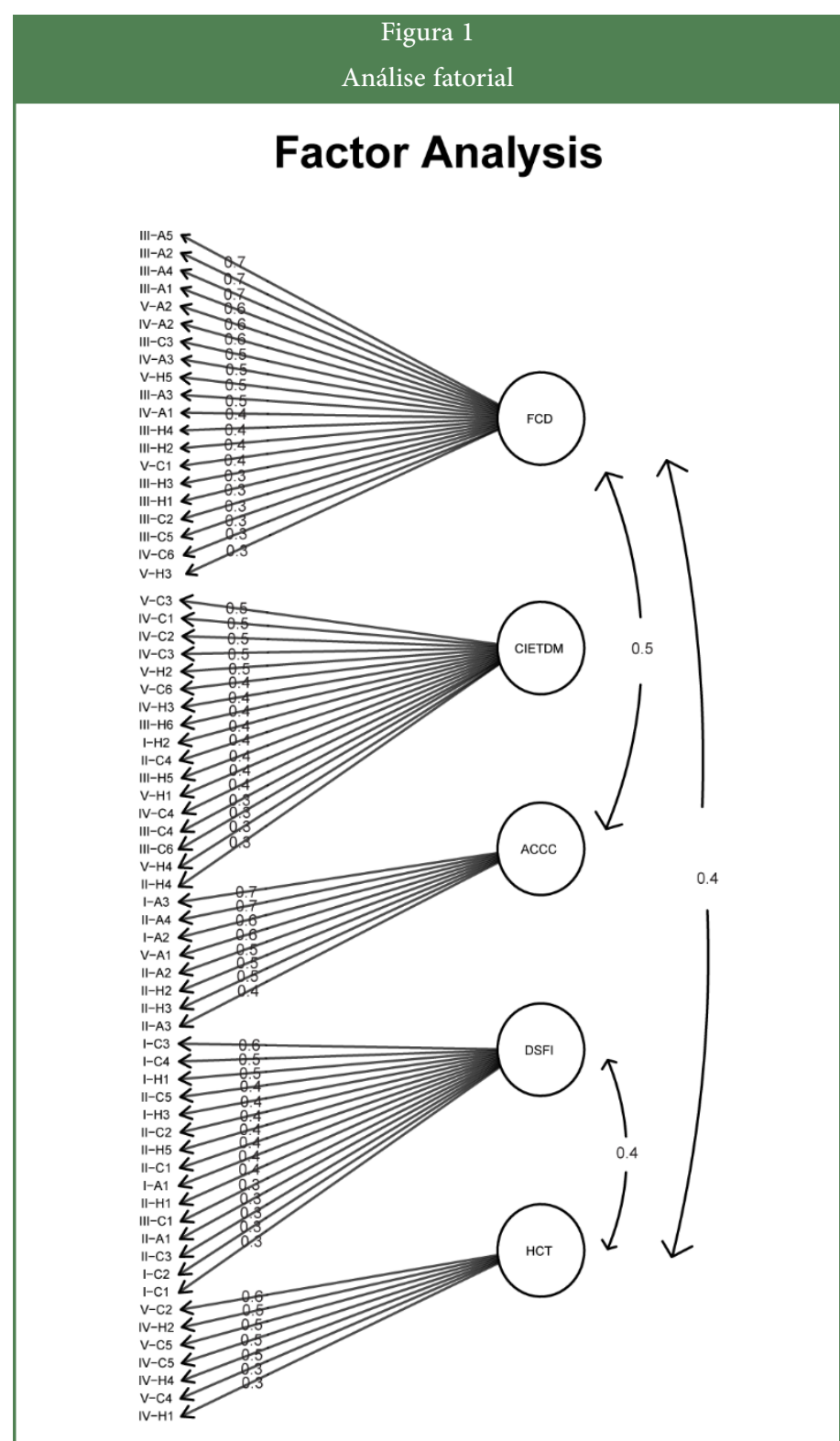

FCD = fundamentação, contexto e definição; ACCC = aspectos-chave da competência cultural; CIETDM = compreendendo o impacto do estereótipo na tomada de decisão médica; DSFI = disparidades em saúde e fatores que a influenciam; HCT = habilidades clínicas transculturais.

Fonte: Elaborada pelos autores.

aprimoramento da competência cultural, pois envolve a identificação das necessidades de diferentes grupos populacionais, de acordo com suas características étnicas, raciais e culturais, no contexto de suas representações sobre o processo saúde-adoecimento-cuidado ${ }^{29}$. Nesse sentido, com a disponibilização do TACCT, considerando-se os objetivos de aprendizagem em relação às abordagens culturais e étnico-raciais, é possível abordar o que deve ser esperado do/a estudante ao fim do curso de Medicina. Os resultados fornecem um panorama geral do currículo de graduação em Medicina, por exemplo, se faltam materiais de apoio que contemplem esses conteúdos ${ }^{30}$. Em suma, os/as estudantes podem avaliar se tiveram contato com esses conteúdos ou não, mensurando todos os conceitos e concepções dos itens dos domínios - que representam o resultado daquilo que se espera de um/a discente que finaliza a graduação 
no curso de Medicina em relação a esses construtos avaliados ${ }^{31}$.

Vale ressaltar que o TACCT foi projetado para fornecer uma visão geral da competência cultural dos currículos nas escolas médicas dos Estados Unidos e, como tal, não se destina a examinar o currículo de competência cultural com um alto nível de especificidade ou a perguntar sobre estratégias de ensino usadas para alcançar os objetivos de aprendizagem nos domínios ${ }^{11}$. Destacamos que o TACCT é uma ferramenta útil para obter uma visão instantânea do currículo de competência cultural, a fim de orientar o processo de identificação de lacunas no ensino e na aprendizagem ${ }^{11}$. Ademais, o TACCT também não foi projetado para abordar o currículo informal, que pode ter uma influência poderosa, mas indeterminada, na aprendizagem dos/as alunos/ as ou na forma como eles/as são avaliados/as ${ }^{11}$.

Um fator favorável para a testagem da tradução e a adaptação transcultural foi o fato de que os/as estudantes tinham um contexto curricular semelhante, o que favoreceu o processo de responder ao TACCT. Além disso, como descrevem Lie, Boker et al. ${ }^{11}$, faltam-nos dados sobre as experiências educacionais dos/as alunos/as entrevistados/as em relação às vivências deles/as antes de ingressarem na Faculdade de Medicina e ao fato de elas terem afetado as respostas dadas. Como o nosso objetivo era testar o entendimento dos itens e as propriedades psicométricas do TACCT, a fim de disponibilizarmos essa matriz para as escolas médicas brasileiras, não consideramos que isso tenha comprometido o trabalho empreendido.

O objetivo do nosso estudo foi estabelecer a viabilidade do uso do TACCT quanto à sua equivalência semântica e às suas propriedades de medida em relação ao instrumento original, a fim de que ele funcione como um norteador para o planejamento curricular nas escolas médicas brasileiras. As análises psicométricas confirmaram a validade dessa matriz de competências, o que possibilitou certificar a sua estrutura fatorial, identificando os itens correspondentes aos cinco domínios. Além disso, a confiabilidade do instrumento foi confirmada.

Examinar as próprias crenças sobre como a medicina é ensinada e praticada é um ponto-chave para entender e aceitar a cultura do paciente ${ }^{31}$. Apenas incluir os temas de maneira longitudinal, como normalmente é proposto em saúde coletiva, será insuficiente se professores/as e estudantes não entenderem que o ponto de partida é uma matriz de competência cultural que garanta o aprendizado que inclui a forma de avaliação do/a estudante para checagem e garantia dessa formação. Isso se faz necessário porque uma boa parcela dos/as estudantes vê a competência cultural como uma ciência que não precisa de treinamento e atribui menor valor a ela do que aos aspectos biológicos da doença ${ }^{32}$.

\section{CONCLUSÃO}

O TACCT é um instrumento que avalia amplamente muitas facetas do ensino sobre competência cultural. A sua versão para o português falado no Brasil é válida e confiável e tem potencial para ser utilizada tanto na fase de implantação de novos conteúdos curriculares quanto no processo de revisão e aprimoramento de currículos das escolas médicas brasileiras.

\section{AGRADECIMENTO}

Agradecemos o fomento da Fundação da UFU do Programa de Graduação (Prograd).

\section{REFERÊNCIAS}

1. Smedley BD. Unequal treatment: confronting racial and ethnic disparities in health care (full printed version). Washington, DC: The National Academies Press; 2003.

2. Wachtler C, Troein M. A hidden curriculum: mapping cultural competency in a medical programme. Med Educ. 2003;37:861-8.

3. Helman, CG. Abrangência da antropologia médica. In: Helman, CG. Cultura, saúde e doença. Tradução Claudia Buchweitz e Pedro Garcez. 4a ed. Porto Alegre: Artmed; 2003.

4. Mayberry RM, Mili F, Ofili E. Racial and ethnic differences in access to medical care. Med Care Res Rev. 2000;57(4 Suppl):108-45.

5. Aeder L, Altshuler L, Kachur E, Barrett S, Hilfer A, Koepfer S, et al. The "Culture OSCE": introducing a formative assessment into a postgraduate program. Educ Health. 2007; 20(1):11-21.

6. Cha-Chi F, Lagha RR, Henderson P, Gomez AG. Working with interpreters: how student behavior affects quality of patient interaction when using interpreters. Med Educ. 2010;15(1):1-7.

7. Parisi V, Ahmed Z, Lardner D, Cho E. Global health simulations yield culturally competent medical providers. Med Educ. 2012; 46(11):1126-7.

8. Betancourt JR, Green AR, Carrillo JE, Ananeh-Firempong O 2nd. Defining cultural competence: a practical framework for addressing racial/ethnic disparities in health and health care. Public Health Rep. 2003;118(4):293-302.

9. Beach MC, Price EG, Gary TL, Robinson KA, Gozu A, Palacio A, et al. Cultural competency: a systematic review of health care provider educational interventions. Med Care. 2005; 43(4):356.

10. Eisenberg L. Disease and illness: distinctions between professional and popular ideas of sickness. Cult Med Psychiatry. 1977;1:9-23.

11. Lie D, Boker J, Cleveland E. Using the Tool for Assessing Cultural Competence Training (TACCT) to measure faculty and medical student perceptions of cultural competence training in the first three years of the curriculum. Acad Med. 2006;81(6):557-64.

12. Boardman J. Critical synthesis package: Tool for Assessing Cultural Competence Training (TACCT). Med Ed Portal. 2015;11:10298.

13. Beaton ED, Bombardier C, Guillemin F, Bosi F M. Guidelines for the process of cross-cultural adaptation of self-report measures. Spine. 2000;25:3186-91.

14. Acquadro C, Katrin C, Asha H, Neil A, European Regulatory Issues and Quality of Life Assessment (ERIQA) Group. Literature review of methods to translate health-related quality of life questionnaires for use in multinational clinical trials. Value Health. 2008;11(3):509-21.

15. Guillemin F, Bombardier C, Beaton D. Cross cultural adaptation of health-related quality of life measures: literature review and proposed guidelines. J Clin Epidemiol. 1993;6:1417-32.

16. Clark LA, Watson D. Constructing validity: basic issues in objective scale development. Psychol Assess. 1995;7(3):309-19.

17. Comrey AL, Lee HB. A first course in factor analysis. 2nd ed. Hillsdale, NJ: Lawrence Erlbaum Associates; 1992.

18. Eremenco SL, Cella D, Arnold BJ. A comprehensive method for the translation and crosscultural validation of health status questionnaires. Eval Health Prof. 2005;28:212-32.

19. Wild D, Grove A, Martin M, Eremenco S, McElroy S, Verjee-Lorenz A, et al. Principles of good practice for the translation and cultural

REVISTA BRASILEIRA DE EDUCAÇÃO MÉDICA

$94(4): \mathrm{e} 168 ; 2020$ 
adaptation process for patient-reported outcomes (PRO) measures: report of the ISPOR Task Force for Translation and Cultural Adaptation. Value Health. 2005;8:94-104.

20. Hasson F, Keeney S, McKenna H. Research guidelines for the Delphi survey technique. J Adv Nurs. 2000;32:1008-15.

21. Hsu C-C, Sandford B. The Delphi technique: making sense of consensus. Pract Assess Res Eval. 2007;12:1-8.

22. Willis GB. Cognitive interviewing: a "how to" guide. 1999 [acesso em $1^{\circ}$ ago 2011]. Disponível em: http://appliedresearch.cancer.gov/ areas/cognitive/interview.pdf.

23. Bonomi AE, Cella DF, Hahn EA, Bjordal K, Sperner-Unterweger $\mathrm{B}$, Gangeri L, et al. Multilingual translation of the Functional Assessment of Cancer Therapy (FACIT) quality of life measurement system. Qual Life Res. 1996;5:309-20.

24. McHorney CA, Ware JE, Lu JFR, Sherbourne CD. The MOS 36 item short-form healthy survey (SF- 36): III. Test of data quality, scaling assumptions and reliability across diverse patient groups. Med Care. 1994;32:40-66.

25. Kuder GF, Richardson MW. The theory of the estimation of test reliability. Psychometrika. 1937;2(3):151-60.

26. Hair JF, Black WC, Babin BJ, Anderson RE, Tatham RL. Multivariate data analysis. 6th ed. New Jersey: Prentice Hall; 2006.

27. Muthen BO, Toit SHC, Spisic D. Robust inference using weighted least squares and quadratic estimating equations in latent variable modeling with categorical and continuous outcomes. Unpublished technical report. 1997; 1-49 [acesso em 16 maio 2020]. Disponível em: http://www.statmodel.com/wlscv.shtml.

28. Brasil. Constituição da República Federativa do Brasil. 35a ed. Brasília: Câmara dos Deputados, Edições Câmara; 2012 [acesso em 17 maio 2020]. Disponível em: http://bd.camara.gov.br/bd/bitstream/ handle/bdcamara/15261/constituicao_federal_35ed.pdf?sequence=9.

29. Starfield B. Atenção primária: equilíbrio entre necessidades de saúde, serviços e tecnologia. Brasília: Unesco, Ministério da Saúde; 2002 [acesso em 17 maio 2020]. Disponível em: http://bvsms.saude.gov.br/ bvs/publicacoes/atencao_primaria_p1.pdf.

30. Shapiro J, Lie D, Gutierrez D, Zhuang G. "That never would have occurred to me": a qualitative study of medical students' views of a cultural competence curriculum. BMC Med Educ. 2006;6(1):1-7.

31. Chun MBJ. Pitfalls to avoid when introducing a cultural competency training initiative. Med Educ. 2010;44(6):613-20.

32. Miller E, Green AR. Student reflections on learning crosscultural skills through a "cultural competence" OSCE. Med Teach. 2007;29(4):76-84.

\section{CONTRIBUIÇÃO DOS AUTORES}

Janaina Carla Silva Oliveira Pimenta, Talitha Nascimento Mendonça, Tânia Maria da Silva Mendonça e Bruno Simão Teixeira realizaram a concepção do estudo, a tradução e adaptação transcultural. Igor Mychael Melo Ferreira, Nathaniel Lucas Soares Lima e Rogério de Melo Costa Pinto realizaram a análise psicométrica e de dados estatísticos. Tânia Maria da Silva Mendonça realizou a supervisão global do estudo.

\section{CONFLITO DE INTERESSES}

Os autores declaram não haver conflito de interesses neste estudo.

\section{ENDEREÇO PARA CORRESPONDÊNCIA}

Janaina Carla Silva Oliveira Pimenta. Rua Luiz Roberto Alcântara, 825, Alto Umuarama, Uberlândia, MG, Brasil. CEP: 38405-355

E-mail: solijanaina@gmail.com

\section{APÊNDICES}

\begin{tabular}{|c|c|c|c|c|c|}
\hline \multicolumn{6}{|c|}{ Apêndice 1} \\
\hline Conceito & Método de Avaliação & Modelo & Ferramenta & Objetivo & Autores \\
\hline Tradução inicial & Tradutor 1 e tradutor 2 & Independentes & $\begin{array}{c}\text { Itens traduzidos para } \\
\text { português falado no Brasil. }\end{array}$ & $\begin{array}{l}\text { A partir do conceito dos itens, } \\
\text { evitar tradução literal. }\end{array}$ & Eremenco, Cella et al. ${ }^{18}$ \\
\hline Reconciliação & $\begin{array}{c}\text { Tradutor especialista na área } \\
\text { de educação }\end{array}$ & $\begin{array}{l}\text { Reconciliação } \\
\text { independente }\end{array}$ & $\begin{array}{c}\text { Versões obtidas na tradução } \\
\text { inicial. }\end{array}$ & $\begin{array}{l}\text { Propor uma tradução alternativa } \\
\text { para garantir a compatibilidade } \\
\text { linguística. }\end{array}$ & Eremenco, Cella et al. ${ }^{18}$ \\
\hline Retrotradução & $\begin{array}{c}\text { Tradutor nativo em inglês e } \\
\text { fluente no português falado } \\
\text { no Brasil }\end{array}$ & $\begin{array}{l}\text { Retrotradução para o } \\
\text { inglês norte-americano }\end{array}$ & $\begin{array}{l}\text { A versão retrotraduzida foi } \\
\text { reconciliada. }\end{array}$ & $\begin{array}{c}\text { Garantir a equivalência de } \\
\text { significado com os itens originais. }\end{array}$ & $\begin{array}{l}\text { Bonomi et al. }{ }^{23} \mathrm{e} \\
\text { Eremenco, Cella et al. }{ }^{18}\end{array}$ \\
\hline Comparação & Coordenador de linguagem & $\begin{array}{l}\text { Comparação da versão } \\
\text { retrotraduzida com a } \\
\text { versão original. }\end{array}$ & $\begin{array}{l}\text { Análise dos conceitos dos } \\
\text { itens e comparação da versão } \\
\text { retrotraduzida. }\end{array}$ & $\begin{array}{c}\text { Analisar discrepâncias entre a } \\
\text { versão retrotraduzida e os itens } \\
\text { originais. }\end{array}$ & $\begin{array}{c}\text { Bonomi et al. }{ }^{23} \mathrm{e} \\
\text { Eremenco, Cella et al. }{ }^{18}\end{array}$ \\
\hline Delphi & Revisores independentes & $\begin{array}{l}\text { Revisão das etapas } \\
\text { anteriores. }\end{array}$ & Selecionar a melhor tradução. & $\begin{array}{l}\text { Propor, se necessário, uma } \\
\text { tradução alternativa. }\end{array}$ & $\begin{array}{c}\text { Hasson, Keeney et al. }{ }^{20} \mathrm{e} \\
\text { Hsu e Sandford }{ }^{21}\end{array}$ \\
\hline Versão pré-final & Coordenador de linguagem & $\begin{array}{l}\text { Análise e comentários } \\
\text { dos revisores. }\end{array}$ & $\begin{array}{c}\text { Eleger a tradução mais } \\
\text { adequada. }\end{array}$ & $\begin{array}{l}\text { Avaliar a equivalência das traduções } \\
\text { com a história dos itens. }\end{array}$ & Eremenco, Cella et al. ${ }^{18}$ \\
\hline Pré-teste & $\begin{array}{l}16 \text { estudantes (dois de cada } \\
\text { período) }\end{array}$ & $\begin{array}{l}\text { Testar a versão pré- } \\
\text { traduzida e adaptada. }\end{array}$ & $\begin{array}{l}\text { Preenchimento do } \\
\text { instrumento, sondagem verbal } \\
\text { e parafraseamento dos itens. }\end{array}$ & Avaliar o entendimento dos itens. & $\begin{array}{c}\text { Willis }^{22} \text { e Eremenco, Cella } \\
\text { et al. }{ }^{18}\end{array}$ \\
\hline $\begin{array}{l}\text { Incorporação do } \\
\text { pré-teste }\end{array}$ & Coordenador de linguagem & Revisão final. & $\begin{array}{l}\text { Analisar as inadequações } \\
\text { percebidas no pré-teste. }\end{array}$ & Gerar a versão final. & $\begin{array}{c}\text { Willis }^{22} \\
\text { e Eremenco, Cella et al. }{ }^{18}\end{array}$ \\
\hline
\end{tabular}

Fonte: Elaborado pelos autores. 


\begin{tabular}{|c|c|c|c|c|c|}
\hline \multicolumn{6}{|c|}{$\begin{array}{l}\text { Apêndice } 2 \\
\text { Análise psicométrica do TACCT }\end{array}$} \\
\hline Conceito & Método de Avaliação & Modelo & Ferramenta & Objetivo & Autores \\
\hline A. Análise de itens & $\begin{array}{l}\text { Frequência de resposta, média, } \\
\text { desvio padrão, amplitude, } \\
\text { assimetria e curtose, dados } \\
\text { perdidos; teste } t \text { independente; } \\
\text { ANOVA One Way. }\end{array}$ & na & SPSS Statistics 24.0 & $\begin{array}{l}\text { Avaliar a qualidade do } \\
\text { conjunto de itens. }\end{array}$ & McHorney, Ware et al. ${ }^{24}$ \\
\hline \multirow[t]{2}{*}{$\begin{array}{l}\text { B. Análise de } \\
\text { escala }\end{array}$} & $\begin{array}{l}\text { Matriz de correlação interitens, } \\
\text { correlações item-escala, coeficiente } \\
\text { de confiabilidade. }\end{array}$ & $\begin{array}{l}\text { Consistência interna do } \\
\text { item; coeficiente KR-20. }\end{array}$ & SPSS Statistics 24.0 & $\begin{array}{l}\text { Avaliar a confiabilidade } \\
\text { dos itens do TACCT. }\end{array}$ & Kuder e Richardson ${ }^{25}$ \\
\hline & $\begin{array}{l}\text { Análise fatorial confirmatória } \\
\text { (AFC) }\end{array}$ & $\begin{array}{c}\text { Medidas de } \\
\text { ajustes (absolutos, } \\
\text { parcimoniosos e } \\
\text { comparativos). }\end{array}$ & $\begin{array}{c}\text { R - Pacotes } \\
\text { Polycor,MASS, } \\
\text { GPArotation e } \\
\text { Parallel }\end{array}$ & $\begin{array}{l}\text { Avaliar a estrutura } \\
\text { fatorial do TACCT. }\end{array}$ & $\begin{array}{l}\text { Hair, Black et al. }{ }^{26} \mathrm{e} \\
\text { Muthen, Toit et al. }{ }^{27}\end{array}$ \\
\hline
\end{tabular}

na $=$ não se aplica.

Fonte: Elaborado pelos autores. 